\title{
MAC ADDRESS ROUTING POLICY OVER THE IP NETWORK
}

\author{
Md. Abdullah Yusuf Imam \\ Assistant Maintenance Engineer \\ Department Of ICT \\ National University \\ Gazipur-1704, Bangladesh
}

\author{
Mr. Prodip Kumar Biswas \\ Sub-Technical Officer \\ Department Of ICT \\ National University \\ Gazipur-1704, Bangladesh
}

\begin{abstract}
Using MAC filtering on a network permits and denies network access to specific devices through the use of blacklists and white lists. In IP networks, the MAC address of an interface can be queried given the IP address using the Address Resolution Protocol (ARP) for internet protocol version 4 (IPV4) or the neighbor discovery protocol (NDP) for IPV6 [1],[2]. In this way, ARP or NDP is used to relate IP addresses (OSI layer 3) to ethernet MAC addresses (OSI layer 2) [3]. A MAC address is like a social security number which remains unchanged for a person's life time (the device), while an IP address is like a postal code which can be changed. Now we find how MAC \& IP are related, how MAC route from pc to switch(Routing scheme).
\end{abstract}

\section{Keywords-Mac, Ip, Route, Network, Address \\ I. WHAT IS A MAC ADDRESS?}

Cunche and Mathieu et al.(2018-08-22) in their work emphasize and addressed that MAC stands for Media Access Control in general. It is set of numbers that identifies network devices. This number is set by the manufacturers and is embedded within the hardware so it cannot be changed. For this reason it is also known as a fixed address [4]. While it was known as an Ethernet address, Wi-Fi and Bluetooth are among the technologies also use MAC addresses.

MAC address can be access through operating system by using commands. IP addresses, MAC addresses are all unique. They all follow the same set of rules when it comes to format [5].

\section{HOW MANY BITS ARE IN A MAC ADDRESS?}

Aaron Mamiit et al (2014-06-12) in their work emphasize and addressed issues of MAC addresses are come up of 12-digit numbers and are made up of 48 bits, or 8 bytes also. Some types of hardware require a 64-bit MAC address [6]. Certain wireless home automation systems may require a 64-bit MAC address. When IPv6 network, the settings change a bit more. These networks translate 48-bit networks to 64-bit networks by inserting an FFFE value in the middle of all. These identifiers are used to differentiate between 48-bit addresses and 64-bit addresses correctly.

\section{WHAT ARE TYPICAL FORMATS FOR MAC ADDRESSES?}

Different formats that are used for MAC addresses, depending upon the network and specific hardware. These addresses are written in the format of MM:MM:MM:SS:SS:SS. This can vary. Two additional formats for 48-bit addresses are MMMM-MM-SS-SS-SS or MMM.MMM.SSS.SSS.

48-bit address that is converted to a 64-bit address would be formatted as MM:MM:MM:FF:FE:SS:SS:SS [7].

\section{WHAT DOES BIT NUMBER OF MAC ADDRESS MEAN?}

Mathy Vanhoef and Matte Célestin and Cunche Mathieu and Cardoso Leonardo and Piessens Frank et al.( 2018-08-22) in their work emphasize and address that the first six digits or 24 bits in a 48-bit network, is a prefix that is used to identify the manufacturer of the device. It is not unusual to find that several devices created by the same manufacturer have different prefix. This is because some of the biggest manufacturers utilize multiple prefixes across their products.

The remaining 24 bits, is essentially a serial number. That identifies the particular device. All products from the same manufacturer that have the same prefix (or first set of 6 digits) will have a distinct second set of numbers. Each will unique. If the prefix is different, the product identifier may be the same, even when the products are from the same manufacturer. It may also find that the identifier is the same between two products from different manufacturers [8]. As already explained, each manufacturer has its own prefix, so this number will be vary. Every device will in some way have a 


\section{International Journal of Engineering Applied Sciences and Technology, 2019 \\ Vol. 3, Issue 11, ISSN No. 2455-2143, Pages 8-11 \\ Published Online March 2019 in IJEAST (http://www.ijeast.com)}

completely unique MAC address, they were made by the same manufacturer or not.

\section{FORMAT OF A MAC ADDRESS}

MAC address is 12-digit ( 6 bytes or 48 bits) hexadecimal numbers. They are usually written in one of the following three formats:

- MM:MM:MM:SS:SS:SS

- MM-MM-MM-SS-SS-SS

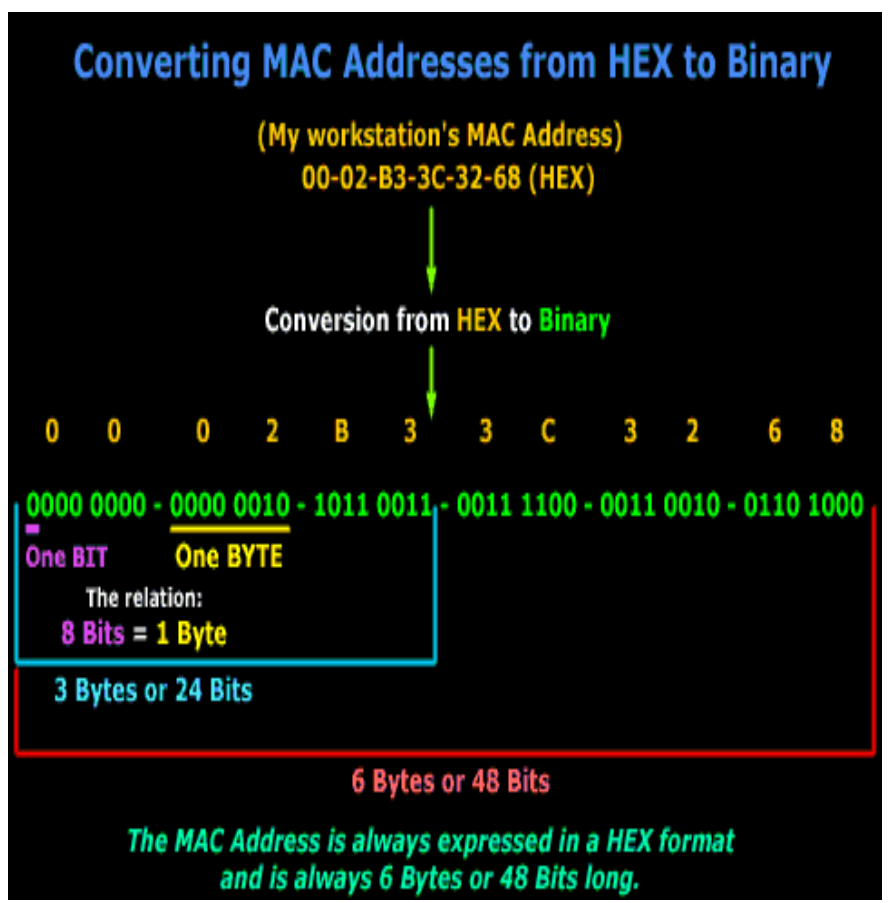

Fig.1. Mac Address translation from Hex to Binary

Leftmost 6 digits (24 bits) called a "prefix" is associated with the adapter manufacturer. Each vendor registers and obtains MAC prefixes as assigned by the IEEE policy. Vendors often possess many prefix numbers associated with their different product. The prefixes 00:13:10, 00:25:9C and 68:7F:74 all belong to Linksys (Cisco Systems) [9].

The rightmost digits of a MAC address represent an identification number for the specific device. All devices manufactured with the same vendor prefix, each is given their own unique 24-bit number. Hardware from different vendors may happen to share the same device portion of the address.

\section{64-BIT MAC ADDRESSES}

Traditional MAC addresses are all 48 bits in length, few types of networks require 64-bit addresses instead. TCP/IP networks based on IPv6 implement a different approach to communicating MAC addresses compared to mainstream IPv4. Instead of 64-bit hardware addresses, though, IPv6 automatically translates 48-bit MAC address to a 64-bit address by inserting a fixed 16-bit value FFFE in between the vendor prefix and the device identifier [10],[11]. IPv6 calls these numbers "identifiers" to distinguish them from true 64bit hardware addresses [12]. Example, a 48-bit MAC address 00:25:96:12:34:56 appears on an IPv6 network as (commonly written in either of these two forms):

- $\quad 00: 25: 96: F F: F E: 12: 34: 56$

- 0025:96FF:FE12:3456

\section{MAC ADDRESS AND DIFFERSNT FUNCTIONS}

Media Access Control Address (MAC address) of a device is a unique identifier assigned to a network interface controller (NIC) for communications at the Data link layer of a network. MAC addresses are used as a network address for most IEEE 802 network technology, including Ethernet.

A MAC may be referred to as the Burned-in Address (BIA). It also be known as an Ethernet Hardware Address (EHA), hardware address or physical address [13].

A network node have multiple NIC and each NIC must has a unique MAC address [14].

Grumbach Emmanuel et al.(2018-08-22) in their work emphasize and addressed the issues also that Address can either Universally Administered Addresses (UAA) or Locally Administered Addresses (LAA). Universally administered address is uniquely assigned to a device by its manufacturer. The first three octets (in transmission order) identify the organization that issue the identifier and is known as the Organizationally unique identifier (OUI). The remain of the address (three octets for EUI-48 or five for EUI-64) are assigned by that organization in nearly any manner they please, subject to the constraint of uniqueness generally. A locally administered address is assigned to a device by a network administrator, overriding the burned-in address process [15].

Universally Administered and Locally Administered Addresses are distinguished by setting the second-leastsignificant bit of the first octet of the MAC address. This bit is called U/L bit, short for Universal/Local, which identifies how the address is administered. If bit is 0, the address is Universally Administered, if it is 1, the address will be 
Locally Administered. Example address 06-00-00-00-00-00 the first octet is 06 (hex), the binary form of which is 00000110 , where the second-least-significant bit is 1 . So, it is a Locally Administered Address [16].

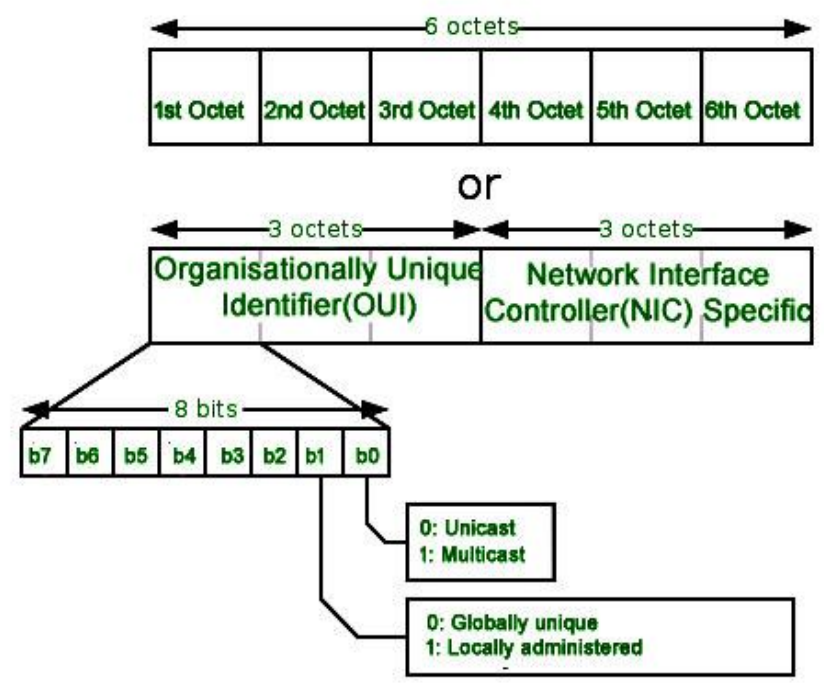

Fig.2. Mac address anatomy

The least significant bit of an address's first octet is 0 (zero), the frame is meant to reach only one receiving NIC. This type of transmission is called Unicast. A Unicast frame is transmitted to all nodes within the collision domain in the switch/router.
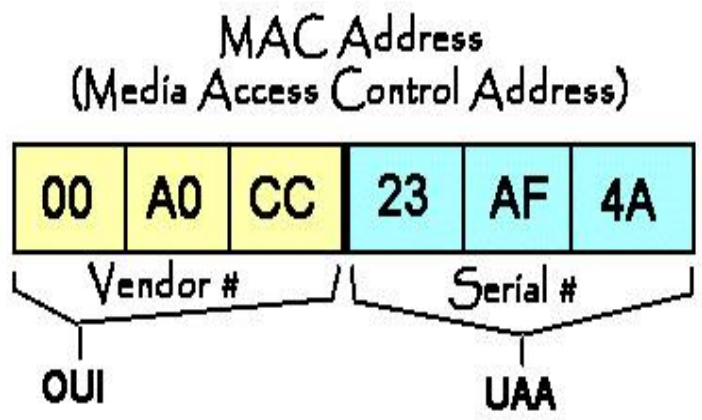

(Organizationally Unique Identifier)

(Universally Administered Address)

Fig.3. Mac address partition

If the least significant bit of the first octet is set to 1 , the frame will still send only once, NICs will choose to accept it based on criteria other than the matching of a MAC address: Example, based on a configurable list of accepted multicast MAC address. This is called Multicast Addressing [17],[18].

While the IP addresses involved indicate the original source and ultimate destination, a MAC address is used only on connections from one piece of networking equipment to the next and so on [19].

\section{MAC ADDRESS ROUTING PROTOCOL}

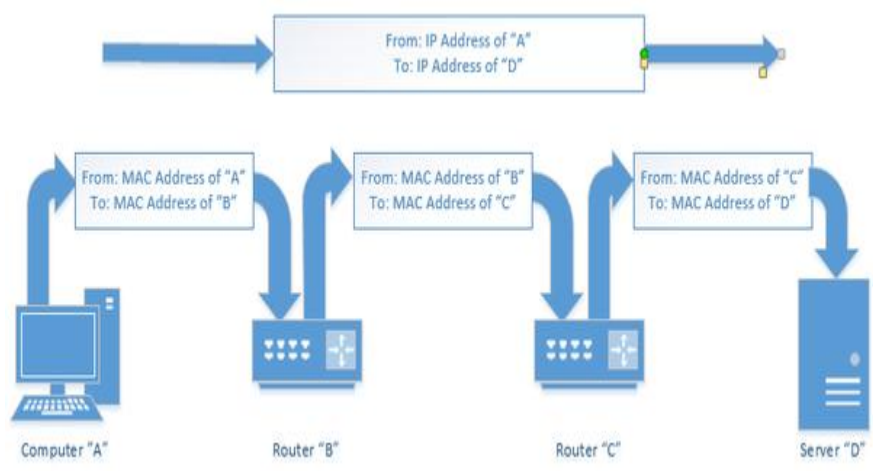

Fig.3. MAC address Routing

When information leaves one computer, it has a computer's network adapter's MAC address. But when it arrives at any router, that MAC address is removed from packet. When the router sends the information further upstream to any ISP's router, it contains the MAC address of that router. When it moves from the ISP's router to another router on the internet, it contains the MAC address of the ISP's last router. And so on.

\section{DECISION:CASE STUDY-}

MAC address is meaningless outside of any local network. It gets stripped out of the network packet by router and router's Mac Address is Route then.

\section{CONCLUSION}

Because MAC unique, MAC addresses can be used to track a man. Cunche, Mathieu et al.(16 October 2016) in their work emphasize and addressed the issues in which he mention when anyone walk around, his smart phone scans for nearby Wi-Fi networks and broadcasts its MAC address.

\section{REFERENCE}

[1] Cisco Retrieved "Configuring Port Security" 14 November 2015

[2] Standards.ieee.org "IEEE -SA- IEEE Registration Authority", Retrieved 2018-09-20. 
[3] Standards.ieee.org "IEEE -SA- IEEE Registration Authority", Retrieved 2018-09-20.

[4] IEEE-SA "Standard Group MAC Addresses: A Tutorial Guide" (PDF), Retrieved 2018-09-20.

[5] IEEE-SA "Guidelines for Fiber Channel Use of the Organizationally Unique Identifier (OUI)" (PDF),Retrieved 2018-10-11.

[6] Support forums.cisco.com "Overview of Layer 2 Switched Networks and Communication | Getting Started with LANs | Cisco Support Community | 5896 | 68421", Retrieved 2016-05-17.

[7] James Bamford "The Most Wanted Man in the World" Wired. p. 4 Retrieved 2014-12-01.

[8] Aaron Mamiit (2014-06-12) "Apple Implements Random MAC Address on iOS 8. Goodbye, Marketers". Tech Times, Retrieved 2014-12-01.

[9] Matte Célestin "Wi-Fi Tracking: Fingerprinting Attacks and Counter-Measures". 2017, Retrieved 2018-08-22.

[10] Mathy Vanhoef and Matte Célestin and Cunche Mathieu and Cardoso Leonardo and Piessens Frank."Why MAC address randomization is not enough: An analysis of Wi-Fi network discovery mechanisms", Retrieved 2018-08-22.

[11] Matte Célestin and Cunche Mathieu and Rousseau Franck and Vanhoef Mathy "Defeating MAC address randomization through timing attacks", Retrieved 2018-08-22
[12] Wang Winkey "Wireless networking in Windows 10". Missing or empty |url= (help)

[13] Grumbach Emmanuel "iwlwifi: mvm: support random MAC address for scanning". Linux commit effd05ac479b, Retrieved 2018-08-22.

[14] Matte Célestin "Wi-Fi Tracking: Fingerprinting Attacks and Counter-Measures". 2017, Retrieved 2018-08-22.

[15] Mathy Vanhoef and Matte Célestin and Cunche Mathieu and Cardoso Leonardo and Piessens Frank "Why MAC address randomization is not enough: An analysis of Wi-Fi network discovery mechanisms". Retrieved 2018-08-22.

[16] Célestin Matte and Cunche Mathieu and Rousseau Franck and Vanhoef Mathy "Defeating MAC address randomization through timing attacks ", Retrieved 2018-08-22.

[17] Cunche, Mathieu "I know your MAC Address: Targeted tracking of individual using Wi-Fi" (PDF). 2013. Retrieved 19 December 2014. 20."ifconfig (8) manual page", Retrieved 16 October 2016.

[18] Security, Stack exchange.com "Hidden network no beacons", Retrieved 16 October 2016.

[19] Configuration Guide for Cisco Secure ACS 4.2.Cisco.February 2008."Agent less Host Configuration Scenario", Retrieved-2015-09 